\title{
TP53 Mutation in Saudi Breast Cancer Patients: Correlations with Cancer Stem Cell Markers Expression and Clinicopathological Variables
}

\author{
Rami Nassir ${ }^{1 *}$, Hanan M. Abd ElMoneim ${ }^{1,2}$, Ghada Esheba ${ }^{1,3}$, Leimia A. Suliman ${ }^{1}$, John Robbins ${ }^{4}$ \\ ${ }^{1}$ Department of Pathology, School of Medicine, Umm Al-Qura University, Makkah, Saudi Arabia \\ ${ }^{2}$ Department of Pathology, Faculty of Medicine, Minia University, Egypt \\ ${ }^{3}$ Department of Pathology, Faculty of Medicine, Tanta University, Egypt \\ ${ }^{4}$ Department of Internal Medicine, University of California-Davis, Davis, California, United States
}

DOI: $\underline{10.36348 / \mathrm{sjpm} .2020 . \mathrm{v} 05 \mathrm{i} 07.001}$

| Received: 11.07.2020 | Accepted: 22.07.2020 | Published: 28.07.2020

*Corresponding author: Dr. Rami Nassir

\section{Abstract}

Background: Breast cancer is one of the most common cancers with a high mortality rate worldwide including Saudi Arabia. It is characterized by the presence of both intra- and inter- tumor heterogeneity. It describes the origin of this heterogeneity to the cancer stem cells (CSC). Breast cancer stem cells are characterized by the expression of the surface markers CD44 and lack or very low expression of CD24. Objectives: To assess cancer stem cell markers (CD44 andCD24) in 126 breast cancer cases by immunohistochemistry and study their association with a different type of TP53 gene mutations by applying next-generation sequencing (NGS). In addition to analyze the association between these markers and the clinicopathological characteristics. Results: CD44 expression was significantly associated with lymph node metastasis, tumor grade, stage (each $\mathrm{P}<0.0001)$, and ER expression $(\mathrm{P}=0.016)$. CD24 expression was found to be associated with lymph node metastasis and ER only. For the molecular analysis, the number of mutations per case was significantly associated with the advanced stage of breast cancer $(\mathrm{p}=0.004)$. Also, missense mutation was highly associated with CD44 and CD24 ( $\mathrm{p}=0.04, \mathrm{p}=0.005)$, respectively. Stop-gained mutation and Frameshift mutation both were associated with CD44 only ( $\mathrm{p}=0.01, \mathrm{p}=0.05)$, respectively. Finally, the synonymous mutation was only associated with the stage of cancer $(\mathrm{p}=0.01)$. Conclusion, we believe that the combination of CD44, CD24, TP53 and TP53 gene mutations can be prognostic factors for breast cancer patients and the information obtained may contribute to the development of a treatment.

Keywords: Breast cancer, Cancer stem cells, CD44, CD24, TP53, Women health.

Copyright @ 2020: This is an open-access article distributed under the terms of the Creative Commons Attribution license which permits unrestricted use, distribution, and reproduction in any medium for non-commercial use (NonCommercial, or CC-BY-NC) provided the original author and sources are credited.

\section{INTRODUCTION}

Breast cancer is the most common malignancy with a high mortality rate in women globally between the age of 20 and 59 [1]. According to the American Cancer Society, the incidence and the mortality of breast cancer varies significantly based on many factors including age, genetic ethnicity, lifestyle, family history, and geographical locations. During the past few years, breast cancer incidence has shown a dramatic increase which was further characterized by the early onset and the aggressiveness of the disease [2]. In addition, several investigations have found that triplenegative breast cancer (TNBC) was characterized by poor prognosis and survival in addition to the ineffectiveness of hormonal therapies [3].

The incidence of breast cancer among Saudi women has increased with a remarkable number of women being affected and diagnosed before the age of
50. This might be explained by the accumulation of genetic factors due to the high incidence of consanguineous marriages leading to breast cancer predisposition. The effects of these genetic factors are probably becoming more evident now due to the social and lifestyle changes brought upon by the relatively recent positive economical upheavals in the country [4, 5]. Furthermore, a high prevalence of breast cancer mortality has been noticed among Saudi women lately. This highlights the very low rate of breast cancer screening and the need to intensify the screening rates in Saudi Arabia [6].

Breast cancer has been found to present intraand inter- tumor heterogeneity, which can occur as genetic, phenotypic or functional diversity in spatial or temporal patterns. The most commonly accepted concepts describing the origin of tumor heterogeneity is the cancer stem cell (CSC). CSCs are capable of high 
proliferation rate, self-renewal, and multidirectional differentiation associated with an important role in tumorigenesis, growth, invasion, metastasis, and recurrence [4]. Minimizing the rate of breast cancer recurrence and improving the rate of the cure in clinical practice has been based on the intense exploration of CSCs $[6,7]$.

Breast cancer stem cells are characterized by the expression of the surface markers CD44 and lack or very low expression of CD24 [8]. The adhesion molecule, CD44, is a cell surface trans-membrane glycoprotein involves in lymphocyte activation, recirculation and homing, adhesion of extracellular matrix, angiogenesis, and cell proliferation and differentiation. These properties are associated with the pathologic activities of cancer cells that were strongly positive for CD44 and negative for CD24, besides having the tumorigenic and metastatic abilities in breast tumor tissue. However, prospective identification, characterization, and isolation of these CSCs have been a major challenge [9]. The CD44 profile showed genes consistent with a stem cell-like profile. Therefore, CD44 and CD24 are important representative CSC biomarkers [10].

Several studies have shown that the overexpression of CD44 promotes tumor invasion and metastasis (20). CD44 appears to have a significant regulatory role in almost all cancer types. The regulatory interaction of CD44 in signaling pathways in tumor cells remains unresolved [9]. However, CD44 plays a major role in the clinical behavior of breast cancer and might be an interesting biomarker for therapeutic targets.

TP53 is the most frequently mutated cancer gene in tumor samples. Somatic mutations in this tumor suppressor gene were found in a wide range of tumors and germline mutations that can cause Li-Fraumeni syndrome, a condition leading to a high, early-onset risk of multiple types of cancer. Evaluation of germline variation in cancer susceptibility genes in a healthy cohort found TP53 missense variants and did not find any nonsense or frameshift variant in 681 individuals [11]. Germline mutations in TP53 may cause an even higher risk of breast cancer. Women who carry germline mutations in the TP53 gene have a very high risk of breast cancer of up to $85 \%$ by age 60 years [12]. In these cases, the mortality occurs due to the development of metastatic or locally recurrent disease resistant to standard chemotherapy and radiation. Increasing evidence suggests that cancer stem-like cells (CSCs), present at diagnosis or evolved during treatment through epithelial-mesenchymal transition (EMT) and/or clonal selection, are the driving force of tumorigenesis, chemo/radio resistance, and metastatic spread [13].

Importantly, the TP53 gene is a tumor suppressor that is not frequently deleted in cancer cases. Instead, this gene primarily sustains various other types of mutations, the most frequent mutation of which is a missense mutation in the TP53 DNA-binding domain. Prevention of the DNA binding process due to the mutation in the TP53 DNA-binding domain dysregulates the induction of TP53-mediated cellular arrest and consequently prevents the apoptotic process [14].

Therefore, the purpose of the current study is to evaluate IHC-detected cancer stem cell markers (CD44 and CD24) and TP53 expression in a cohort of 126 patients with breast cancer, and to evaluate the association between CD44, CD24, TP53 expression and the prognostic clinicopathological variables. In addition, we will determine the type of TP53 mutation by applying whole-exome sequencing with a spectrum of germline TP53 mutations of 23 cases diagnosed with TNBC based on TP53 immunohistochemical expression.

\section{MATERIAL AND METHODS \\ Patients and Tissue Samples}

The current study was approved by the ethics committee at the school of medicine at Umm Al-Qura University. Tissue samples were consecutively recruited between 2015 and 2019 from local hospitals in the western provenance of Saudi Arabia for 126 cases diagnosed with breast cancer. All hematoxylin/eosin (H\&E) stained sections were reevaluated to confirm the histopathological type. Clinical and pathological information, including age, tumor size, type of tumor, disease stage, axillary lymph nodes, tumor recurrences, regional and distant metastasis, treatment, disease-free survival (DFS), and overall survival (OS) were collected, accordingly (Table-1). We excluded any patients who had undergone radiotherapy, chemotherapy, targeted therapy, or adjuvant endocrine treatment prior to surgery. 
Table-1: Clinicopathological features of breast cancer patients included in this study

\begin{tabular}{|c|c|c|c|}
\hline \multicolumn{2}{|c|}{ Clinicopathological Features } & $\mathbf{N}$ & $\%$ \\
\hline \multirow{2}{*}{ Age } & $\leq 50$ & 52 & $41.3 \%$ \\
\hline & $>50$ & 74 & $58.7 \%$ \\
\hline \multirow{3}{*}{ Size } & less2 & 40 & $31.7 \%$ \\
\hline & $2-5$ & 64 & $50.8 \%$ \\
\hline & More than 5 & 22 & $17.5 \%$ \\
\hline \multirow{2}{*}{ Necrosis } & Absent & 68 & $54.0 \%$ \\
\hline & Present & 58 & $46.0 \%$ \\
\hline \multirow{3}{*}{ Type of Tumor } & IDC & 90 & $71.4 \%$ \\
\hline & other Invasive & 33 & $26.2 \%$ \\
\hline & DCIS & 3 & $2.4 \%$ \\
\hline \multirow{3}{*}{ Nodal State } & Negative & 23 & $18.3 \%$ \\
\hline & Positive $(<3)$ & 65 & $51.6 \%$ \\
\hline & Positive $(>3)$ & 38 & $30.2 \%$ \\
\hline \multirow{3}{*}{ Grade } & Grade 1 & 27 & $21.4 \%$ \\
\hline & Grade 2 & 61 & $48.4 \%$ \\
\hline & Grade 3 & 38 & $30.2 \%$ \\
\hline \multirow{4}{*}{ Stage } & Stage 1 & 13 & $10.3 \%$ \\
\hline & Stage 2 & 70 & $55.6 \%$ \\
\hline & Stage 3 & 36 & $28.6 \%$ \\
\hline & Stage4 & 7 & $5.6 \%$ \\
\hline \multirow{2}{*}{ CD44 } & Negative & 49 & $38.9 \%$ \\
\hline & Positive & 77 & $61.1 \%$ \\
\hline \multirow{2}{*}{ CD24 } & Negative & 69 & $54.8 \%$ \\
\hline & Positive & 57 & $45.2 \%$ \\
\hline \multirow{2}{*}{ ER } & Negative & 47 & $37.3 \%$ \\
\hline & Positive & 79 & $62.7 \%$ \\
\hline \multirow{2}{*}{ PR } & Negative & 53 & $42.1 \%$ \\
\hline & Positive & 73 & $57.9 \%$ \\
\hline \multirow{2}{*}{ HER } & Negative & 91 & $72.2 \%$ \\
\hline & Positive & 35 & $27.8 \%$ \\
\hline \multirow{2}{*}{ TP53 } & Negative & 59 & $46.8 \%$ \\
\hline & Positive & 67 & $53.2 \%$ \\
\hline TNBC & ------ & 23 & $18.3 \%$ \\
\hline Non TNBC & ------ & 103 & $81.7 \%$ \\
\hline
\end{tabular}

LVI: lymphovascular invasion, TNBC: triple negative breast cancer

IDC was graded according to the modification of Elston Ellis on Bloom and Richardson grading system [15]. Specimens were classified based on TNM classification, according to the 2003 WHO classification of breast tumors [16]. A case was considered positive for ER and PR protein expression if more than $15 \%$ of the tumor cells showed nuclear staining [17]. HER2 expression was scored according to the guidelines of the American Society of Clinical Oncology/College of American Pathologists [18].

\section{Immunohistochemistry}

Following deparaffinization and dehydration in hydrogen peroxide, the tissue sections were incubated at $37^{\circ} \mathrm{C}$ for one hour with anti- CD44, CD24, and TP53 mouse monoclonal antibody. Corresponding biotinylated anti- IgG was added and incubated for 30 $\min$ at $37^{\circ} \mathrm{C}$. Then, the sections were incubated with 3,3'- diaminobenzidine (DAB) substrate chromogen solution and counterstained with hematoxylin. Negative controls were incubated with phosphate-buffered saline (PBS) instead of the primary antibody. Known positive tissues were used as positive controls.

All immunohistochemical markers were given a positive or negative score; negative cases were those that displayed no staining or staining in less than a certain percentage of tumor cells, and positive cases were those with unequivocal staining in at least a certain percentage of tumor cells. For CD24, cases in which it was expressed in $<10 \%$ of cell nuclei and cell membrane were considered negative, whereas those in which CD24 was present in $>10 \%$ of cells were considered positive. CD44 staining was detected mainly in the membrane and the scoring was done in the same manner as CD24 [19]. The expression of TP53 is localized in the nucleus and observation of $\geq 10 \%$ of positive cells was considered TP53 positive [3].

\section{Tumor Dissection and DNA Extraction}

Based on evaluating and so as to achieve a more homogeneous study population, we ended up with 
67 samples qualified for further molecular analysis. However, those 67 breast cancer patients with positive and high expression of TP53 protein including patients with triple-negative subtype breast TNBC (21 cases) and Non-TNBC (46 cases). From each selected case, 10 -nm-thick tissue sections obtained from paraffinembedded samples were collected in the Eppendorf safe-lock tube to be prepared for DNA extraction. The DNA was extracted from the tissue collected using the QIAamp DNA FFPE Tissue Kit (Qiagen, Hilden, Germany) according to the protocol of the manufacture. The extracted DNA was eluted into $40 \mathrm{~nL}$ of buffer, quantified using two methods: NanoDrop microvolume sample retention system (Thermo Fisher Scientific NanoDrop Products, Hanover Park, IL, USA). The second method of quantification was using Qubit, DS (Thermo Fisher Scientific, Waltham, MA, USA) and stored at $4^{\circ} \mathrm{C}$. Of which 48 cases were fulfilled the recommended DNA quality required for the molecular analysis.

\section{Next Generation Sequencing}

In all 48 cases, 20ng of DNA extracted was prepared for sequencing. The assessment of 15 genes (AKT1, GNA11, NRAS, BRAF, GNAQ, PDGFRA, EGFR, KIT, PIK3CA, ERBB2, KRAS, RET, FOXL2, MET and TP53) was applied using TruSight Tumor 15 (Illumina, San Diego, CA, USA). The resulting pooled libraries were quality controlled using The Qubit ${ }^{\circledR}$ dsDNA HS (High Sensitivity) Assay Kits make DNA quantitation easy and accurate. Sequencing was performed with paired-end reads on the MiSeq Platform (Illumina ${ }^{\circledR}$ ).

\section{Detection of Variations}

The sequenced reads were arranged and compared to the hg19/GRCh37 reference sequence and analyzed using the MiSeq reporter (Illumina). To identify variants in breast tissue samples, BaseSpcae Variant Interpreter (Illumina) was applied. The called variants were considered somatic malignant tumors of the breast (SNOMEDCT) version 4.0.7.6. To reduce the false-positive rate, we set the cutoff value as follows: read depth $>100$, Indel repeat length <8; allele frequency of mutant reads $>1 \%$, genotype quality $>30$.

\section{STATISTICAL ANALYSIS}

Association between CD44, CD24, TP53 protein expression pattern, TP53 gene polymorphism, and the clinicopathological parameters were assessed by Chi-square statistical test and Pearson correlation. Survival analyses were estimated using the KaplanMeier method and compared using the log-rank test. Cox proportional hazards model was used to estimate the hazard ratio (HR) of each clinicopathological variables for OS and DFS. All predictors with P-value $<0.05$ in univariate Cox analyses were used in multivariate analysis. P-values were 2-tailed and considered significant when $<0.05$. Also, $X^{2}$ tests were applied to test the association of different types of TTP53 mutations with CD44, CD24, and other risk variables for breast cancer. Statistical analyses were carried out using SPSS statistics 22.0 software.

\section{RESULTS \\ Patient Characteristics}

One hundred and twenty-six cases were included in this study. The clinicopathological characteristics of the patients were described in detail in Table-1. Briefly, the mean age was $54.7 \pm 12.9$ years (29-87 years). Forty patients $(31.7 \%)$ developed recurrence and forty-one patients $(32.5 \%)$ died by the end of follow-up. Twenty-three cases (18.3\%) had negative lymph nodes and nearly half of the cases (55.6\%) had stage II disease. Triple-negative breast cancer (TNBC) cases were 23/126 (18.3\%) while 103 $(81.7 \%)$ were non triple-negative breast cancer cases, and those include luminal $\mathrm{A}$, luminal $\mathrm{B}$, and HER/2 positive cases.

\section{Correlation of CD44 and CD24 Expressions with Clinicopathological Variables:}

Correlation of CD44 and CD24 expressions with the different clinicopathological variables are summarized in Table-2. CD44 was expressed on the cell membranes of tumor cells in 77 cases $(61.1 \%$ ) (Fig1A). CD44 expression was significantly associated with lymph node metastasis, tumor grade, stage (each $\mathrm{P}<0.0001)$, and ER expression $(\mathrm{P}=0.016)$. Notably, the expression of CD44 was found to be positively associated with the age; however, this association was not statistically significant $(\mathrm{p}=0.076)$. On the other hand, cytoplasmic and membranous staining for CD24 was detected in 57 cases (45.2\%) (Fig.1B). CD24 expression was found to be associated with lymph node metastasis and ER, however, this association was not statistically significant $(\mathrm{P}=0.055$ and $\mathrm{P}=0.079$ respectively). 
Table-2: Association between CD24 and CD44 expression and clinicopathological characteristics in breast cancer

\begin{tabular}{|c|c|c|c|c|c|c|c|c|c|c|c|}
\hline \multirow{3}{*}{\multicolumn{2}{|c|}{$\begin{array}{l}\text { Clinicopathological } \\
\text { Features }\end{array}$}} & \multicolumn{4}{|c|}{ CD44 } & \multirow{3}{*}{$\begin{array}{l}\text { * } \mathrm{P}- \\
\text { value }\end{array}$} & \multicolumn{4}{|c|}{ CD24 } & \multirow{3}{*}{$\begin{array}{l}{ }^{*} \mathrm{P}- \\
\text { value }\end{array}$} \\
\hline & & \multicolumn{2}{|c|}{ Negative } & \multicolumn{2}{|c|}{ Positive } & & \multicolumn{2}{|c|}{ Negative } & \multicolumn{2}{|c|}{ Positive } & \\
\hline & & $\mathrm{N}$ & $\%$ & $\mathrm{~N}$ & $\mathrm{~N} \%$ & & $\mathrm{~N}$ & $\%$ & $\mathrm{~N}$ & $\%$ & \\
\hline \multirow[t]{2}{*}{ Age } & $\leq 50$ & 21 & $36.8 \%$ & 31 & $44.9 \%$ & \multirow[t]{2}{*}{0.076} & 26 & $36.6 \%$ & 26 & $47.3 \%$ & \multirow[t]{2}{*}{0.206} \\
\hline & $>50$ & 36 & $63.2 \%$ & 38 & $55.1 \%$ & & 45 & $63.4 \%$ & 29 & $52.7 \%$ & \\
\hline \multirow[t]{3}{*}{ Size } & $<2$ & 22 & $38.6 \%$ & 18 & $26.1 \%$ & \multirow[t]{3}{*}{0.501} & 20 & $28.2 \%$ & 20 & $36.4 \%$ & \multirow[t]{3}{*}{0.205} \\
\hline & $2-5$ & 29 & $50.9 \%$ & 35 & $50.7 \%$ & & 41 & $57.7 \%$ & 23 & $41.8 \%$ & \\
\hline & $>5$ & 6 & $10.5 \%$ & 16 & $23.2 \%$ & & 10 & $14.1 \%$ & 12 & $21.8 \%$ & \\
\hline \multirow[t]{2}{*}{ Necrosis } & Absent & 22 & $44.9 \%$ & 46 & $59.7 \%$ & \multirow[t]{2}{*}{0.103} & 33 & $47.8 \%$ & 35 & $61.4 \%$ & \multirow[t]{2}{*}{0.128} \\
\hline & Present & 27 & $55.1 \%$ & 31 & $40.3 \%$ & & 36 & $52.2 \%$ & 22 & $38.6 \%$ & \\
\hline \multirow{3}{*}{$\begin{array}{l}\text { Nodal } \\
\text { State }\end{array}$} & Negative & 16 & $32.7 \%$ & 7 & $9.1 \%$ & \multirow[t]{3}{*}{$<0.0001$} & 16 & $23.2 \%$ & 7 & $12.3 \%$ & \multirow[t]{3}{*}{0.055} \\
\hline & Positive $<3$ & 26 & $53.1 \%$ & 39 & $50.6 \%$ & & 29 & $42.0 \%$ & 36 & $63.2 \%$ & \\
\hline & Positive > 3 & 7 & $14.3 \%$ & 31 & $40.3 \%$ & & 24 & $34.8 \%$ & 14 & $24.6 \%$ & \\
\hline \multirow[t]{3}{*}{ Grade } & Grade 1 & 22 & $44.9 \%$ & 5 & $6.5 \%$ & \multirow[t]{3}{*}{$<0.0001$} & 15 & $21.7 \%$ & 12 & $21.1 \%$ & \multirow[t]{3}{*}{0.989} \\
\hline & Grade 2 & 25 & $51.0 \%$ & 36 & $46.8 \%$ & & 33 & $47.8 \%$ & 28 & $49.1 \%$ & \\
\hline & Grade 3 & 2 & $4.1 \%$ & 36 & $46.8 \%$ & & 21 & $30.4 \%$ & 17 & $29.8 \%$ & \\
\hline \multirow[t]{4}{*}{ Stage } & Stage 1 & 6 & $12.2 \%$ & 7 & $9.1 \%$ & \multirow[t]{4}{*}{$<0.0001$} & 6 & $8.7 \%$ & 7 & $12.3 \%$ & 0.557 \\
\hline & Stage 2 & 38 & $77.6 \%$ & 32 & $41.6 \%$ & & 36 & $52.2 \%$ & 34 & $59.6 \%$ & \\
\hline & Stage 3 & 5 & $10.2 \%$ & 31 & $40.3 \%$ & & 22 & $31.9 \%$ & 14 & $24.6 \%$ & \\
\hline & Stage4 & 0 & $0.0 \%$ & 7 & $9.1 \%$ & & 5 & $7.2 \%$ & 2 & $3.5 \%$ & \\
\hline CD44 & Negative & 49 & $100.0 \%$ & 0 & $0.0 \%$ & & 24 & $34.8 \%$ & 25 & $43.9 \%$ & 0.298 \\
\hline & Positive & 0 & $0.0 \%$ & 77 & $100.0 \%$ & & 45 & $65.2 \%$ & 32 & $56.1 \%$ & \\
\hline CD24 & Negative & 24 & $49.0 \%$ & 45 & $58.4 \%$ & 0.298 & 69 & $100.0 \%$ & 0 & $0.0 \%$ & \\
\hline & Positive & 25 & $51.0 \%$ & 32 & $41.6 \%$ & & 0 & $0.0 \%$ & 57 & $100.0 \%$ & \\
\hline ER & Negative & 22 & $44.9 \%$ & 25 & $32.5 \%$ & 0.016 & 21 & $30.4 \%$ & 26 & $45.6 \%$ & 0.079 \\
\hline & Positive & 27 & $55.1 \%$ & 52 & $67.5 \%$ & & 48 & $69.6 \%$ & 31 & $54.4 \%$ & \\
\hline PR & Negative & 20 & $40.8 \%$ & 33 & $42.9 \%$ & 0.821 & 27 & $39.1 \%$ & 26 & $45.6 \%$ & 0.463 \\
\hline & Positive & 29 & $59.2 \%$ & 44 & $57.1 \%$ & & 42 & $60.9 \%$ & 31 & $54.4 \%$ & \\
\hline HER & Negative & 33 & $67.3 \%$ & 58 & $75.3 \%$ & 0.330 & 48 & $69.6 \%$ & 43 & $75.4 \%$ & 0.464 \\
\hline & Positive & 16 & $32.7 \%$ & 19 & $24.7 \%$ & & 21 & $30.4 \%$ & 14 & $24.6 \%$ & \\
\hline TP53 & Negative & 25 & $51.0 \%$ & 34 & $44.2 \%$ & 0.470 & 36 & $52.2 \%$ & 23 & $40.4 \%$ & 0.212 \\
\hline & Positive & 24 & $49.0 \%$ & 43 & $55.8 \%$ & & 33 & $47.8 \%$ & 34 & $59.6 \%$ & \\
\hline TNBC & & 9 & $39.1 \%$ & 14 & $60.9 \%$ & 0.979 & 10 & $43.5 \%$ & 13 & $56.5 \%$ & 0.229 \\
\hline Non TNBC & & 40 & $38.8 \%$ & 63 & $61.2 \%$ & & 59 & $57.3 \%$ & 44 & $42.7 \%$ & \\
\hline
\end{tabular}

*Statistically significant $\mathrm{P}$-values $(\mathrm{P}<0.05)$ are highlighted in bold.

\section{Correlation of TP53 Expression with Clinicopathological Variables:}

The expression ratio of TP53 in 126 specimens was $53.1 \%$ (67/126) (Fig.1C). TP53 expression was associated with lymphovascular invasion $(68.4 \%$ vs $31.6 \%, \mathrm{P}=0.03)$ and higher histologic grade $(\mathrm{G} 1$ vs $\mathrm{G} 2$ vs $\mathrm{G} 3,44.4 \%$ vs $45.9 \%$ vs $71.1 \%$, respectively; $\mathrm{P}=0.03)$. Significantly, TP53 expression was highest in the G3 group $(71.1 \%)$. The frequency of TP53 expression being lowest in the Non -TNBC and highest in the TNBC cases $21 / 23(91.3 \%, \mathrm{P}<0.0001)$.

\section{Different TP53 mutations detection using Next Generation Profile}

We purified DNA from 48 breast cancer cases from contiguous areas of tissues to be sequenced by the next-generation sequencing method. These 48 samples were sequenced, and of which there was either complete failure or very low quality in 22 samples due to poor DNA quality (read depth <30; alternative variant frequency $<5$ ). We believe the DNA has been degraded in the FFPE material and therefore these cases were excluded from further analysis. The remaining 26 samples were successfully sequenced. Three cases showed negative TP53 mutation, which led to being excluded from the current study. The mean count of the total aligned reads for each sample was 2.7 million (Range 1.9-4.2 million) and the minimum sequencing depth was $484.5 \mathrm{X}$. A number of steps were applied to filter the variations identified in the screening. Any intronic variants were excluded then and variants without pathogenic relevance were removed. This led us to 28 genetic variants and all these 28 variants were detected with known or likely pathogenic effect.

A total of 1368 variants were excluded from the study because they were known polymorphisms, likely non-pathogenic, or did not pass the quality criteria. The number of genes mutations per sample ranged from one to nine mutations with a median of five. Fourteen samples of the 23 samples showed only a single mutation $(60.8 \%)$ and the rest of the samples showed a range from two mutations or more (up to nine mutations per one sample). These 28 variants consisted of 20 missense mutation (71.4\%), five-stop gained mutation $(17.9 \%)$, two synonymous mutations $(7.1 \%)$, and one frameshift mutation (3.6\%). In our cohort study, most of the sequenced samples showed multivariations each with unique molecular profiles. 
Association of the TP53 gene polymorphism with the clinicopathological features in breast cancer cases

To study the association of TP 53 gene polymorphism on breast cancer stem cells, we applied Pearson Chi-Square tests with different clinicopathological variables (Table-1). The number of mutations per case was significantly associated with the stage of breast cancer $(\mathrm{p}=0.004)$. However, number of the mutations was not identified to be significantly associated with other variables.

The most common mutation in our sample set was a missense mutation $(71.4 \%)$ followed by stop gained mutation $(17.9 \%)$ then synonymous mutation $(7.1 \%)$ and one case with a frameshift mutation (3.6 $\%)$. For further analysis, we applied Pearson ChiSquare tests to study the association of the type of mutation with stem cell markers. A missense mutation was highly associated with CD44 and CD24 ( $p=0.04$, $\mathrm{p}=0.005)$, respectively. Stop-gained mutation and Frameshift mutation were both associated with CD44 only $(p=0.01, p=0.05)$, respectively. Finally, the synonymous mutation was only associated with the stage of cancer $(\mathrm{p}=0.01)$.
Correlation of TP53, CD44 and CD24 expressions with overall survival

The mean follow-up time was $3 \pm 1.1$ years ( $1-$ 5 years). Univariate and multivariate analyses of overall survival were summarized in Table 3. In univariate survival analysis, patients with negative TP53 expression had a significantly better overall survival; (HR 2.3; 95\% CI [1.19- 4.57], $\mathrm{p}=0.014)$ ) (Fig. 2C). However, TP53 expression was not an independent prognostic factor in multivariate analysis $(\mathrm{HR}=1.433$; $95 \%$ CI [0.905- 2.270], $\mathrm{p}=0.125)$. In univariate survival analysis, patients with negative CD44 expression had a significantly better overall survival; (HR 18.97; 95\% CI [4.57- 78.73], p<0.0001) (Fig. 2A). Furthermore, CD44 expression was an independent prognostic factor in multivariate analysis $(\mathrm{HR}=8.299$; 95\% CI [1.823- 37.782], $\mathrm{p}=0.006)$. In contrast to CD44, no significant association has been found between positive CD24 expression and overall survival; (HR 0.911; 95\% CI [0.49- 1.68], p=0.76), (Fig. 2B). Furthermore, CD24 expression was not an independent prognostic factor in multivariate analysis $(\mathrm{HR}=1.856$; 95\% CI [0.872- 3.949], p =0.109). In multivariate analysis; high tumor grade, advanced stage, and positive CD44 expression have been proven to be independent negative prognostic factors for OS ( $\mathrm{p}=$ $0.002, \mathrm{p}=0.025$, and $\mathrm{p}=0.006$ respectively).

Table-3: Univariate and multivariate analyses of overall survival according to clinical, histopathological, CD44 and CD24 expressions

\begin{tabular}{|c|c|c|c|c|c|c|c|c|}
\hline \multicolumn{5}{|c|}{ Univariate } & \multicolumn{4}{|c|}{ Multivariate } \\
\hline & \multirow[t]{2}{*}{$\operatorname{Exp}(B)$} & \multirow[t]{2}{*}{ Sig. } & \multicolumn{2}{|c|}{$95.0 \% \mathrm{CI}$ for $\operatorname{Exp}(\mathrm{B})$} & \multirow[t]{2}{*}{$\operatorname{Exp}(B)$} & \multirow[t]{2}{*}{ Sig. } & \multicolumn{2}{|c|}{$95.0 \% \mathrm{CI}$ for $\operatorname{Exp}(\mathrm{B})$} \\
\hline & & & Lower & Upper & & & Lower & Upper \\
\hline CD44 & 18.968 & 0.000 & 4.570 & 78.734 & 8.299 & 0.006 & 1.823 & 37.782 \\
\hline CD24 & 0.911 & 0.766 & 0.493 & 1.684 & 1.856 & 0.109 & 0.872 & 3.949 \\
\hline P53 IHC & 2.334 & 0.014 & 1.190 & 4.577 & 1.338 & 0.472 & 0.605 & 2.956 \\
\hline
\end{tabular}

HR: Hazard ratio, CI: Confidence interval.

Statistically significant $P$-values $(\mathbf{P}<0.05)$ are highlighted in bold.
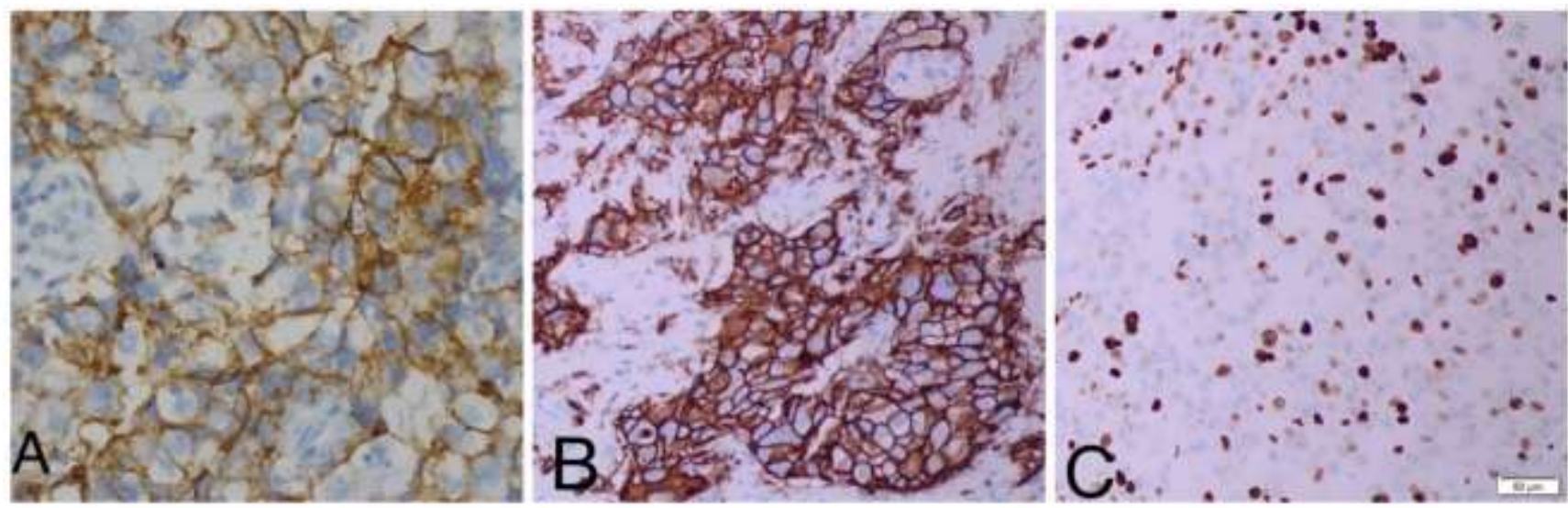

Fig-1: Expression of CD44, CD24, and TP53: A: membranous staining for CD44 (IHC x 400), B: membranous and cytoplasmic staining for CD24 (IHC x 200), C: nuclear staining for TP53 (IHC x 200) 

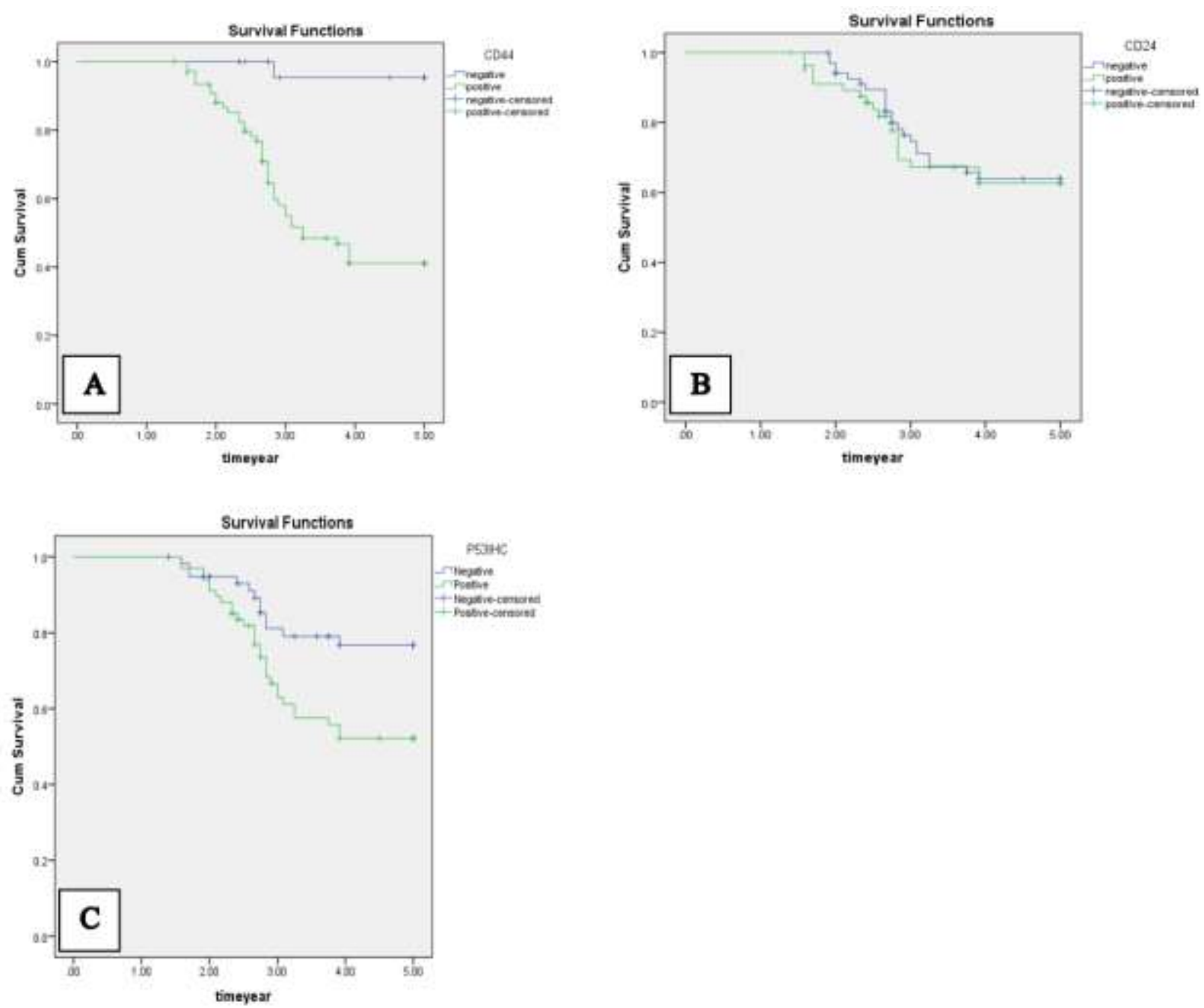

Fig-2: Kaplan-Meier survival curves for overall survival according to the expression of CD44, CD24, and TP53 in tumor cells. (A) CD44 expression, (B) CD24 expression, (C) TP53 expression Patients with negative CD44 and TP53 expressions achieved a significant better OS

\section{DISCUSSION}

An increasing number of studies have shown that an enlarged cancer stem cell population is highly associated with tumor metastatic ability and cancer stem cells can be extended to drive cancer progression. Although there is a degree of consensus on the use of certain CSC markers, some controversy persists about the need to identify further markers [7]. In the current study, we found a strong significant correlation between CD44 expression and histological grade, lymph node metastasis, and stage of the tumor $(\mathrm{P}<0.0001)$. A finding which led us to suggest that positive CD44 expression may play a role in tumor aggressiveness. Similar results have been previously reported. On the contrary, Chen et al could not find any significant association between CD44 expression and the different clinicopathological variables except for ER and PR status [20]. Our study, in particular, demonstrated that CD44 was highly expressed in both TNBC and nonTNBC, however, it was higher in TNBC specifically comparing to non-TNBC. Notably, cancer stem cell markers, especially CD44, might be an interesting target for developing new therapeutic strategies, particularly for TNBC. Similar results were obtained by
Bai et al., and Idowu et al., [21, 22]. In the current study, we found that patients with negative CD44 expression had a significantly better overall survival in univariate analysis. Additionally, CD44 expression was an independent prognostic factor in multivariate analysis. Similar to our results, previous studies showed that the overexpression of CD44 is evidence of worse clinical behavior and shorter progression-free survival [20, 23, 13, 24].

In our study, CD24 expression was found to be associated with lymph node metastasis and ER, however, this association did not reach statistical significance. This finding suggests that CD24 is associated with the aggressive phenotype of breast cancer cells. These results were consistent with a previous study that concluded that higher expression of CD24 was correlated with more advanced histological grade of breast tumors and lymph node involvement [25]. Similarly, Chen et al reported that CD24 expression was not significantly associated with tumor characteristics, however, it was significantly associated with other poor prognostic variables such as negative hormone receptors and positive HER2 and triple- 
negative (TN) phenotype [20]. We could not find any significant association between CD24 expression and overall survival. Furthermore, CD24 expression was not an independent prognostic factor in multivariate analysis. Therefore, our data suggest that that CD24 is associated with tumorigenesis in breast cancer, however, it is not a sufficient factor to determine the prognosis of invasive breast cancer. Similarly, Jang et $a l$, found no difference in clinical outcome and survival with respect to CD24 expression [26]. In contrast to our results, a study done by Wang et al., [27] claimed that CD24 was significantly associated with worse survival. In particular, CD24 may play a role in tumorigenesis and cancer progression [27]. Larger well-designed prospective cohort studies are needed to further evaluate the association between CD24 and the survival of patients with breast cancer.

In our research, TP53 IHC has provided enormous benefits in selecting only cases with the positive expression for gene sequencing in order to achieve a more homogeneous study population. In the current study, TP53 expression was found to be associated with lymphovascular invasion and high histologic grade. This is consistent with other studies that outlined the role of TP53 in the progression of breast carcinoma and indicated a poor prognosis [3, 28]. The high frequency of TP53 expression in the TNBC is an independent prognostic factor, which might aid in identifying subgroups of TNBC patients who are more likely to have a poor outcome and to whom specific therapies might be directed [29, 3]. In our study detected TP53 protein expression by immunohistochemistry reflected the mutant TP53 gene and we found that negative TP53 expression was a favorable prognostic indicator for better overall survival, however, it was not an independent prognostic factor in the multivariate analysis. Similar results have been previously reported [29, 3, 28].

In this study, the majority of TP53 mutations were missense mutations $(71.4 \%)$. This is also consistent with the other studies, most of the TP53 mutations were missense mutation found in the DNA binding domain [30]. In vivo models highlight the aggressive nature of TP53 somatic mutations and the parallel tumor evolution pattern driven by a TP53 missense mutation as the initiating event. In addition, TP53 mutations are the most frequent genetic alterations in breast cancer and are associated with more aggressive disease and worse overall survival [31]. These results were in keeping with previously published data which have shown that mutant p53 enhances tumor development which involves the expansion of CSCs sub-populations within these tumors and that mutant p53 facilitates the acquisition of CSCs phenotype [32].

Our study has several important limitations. Although this study is the first to examine the effect of the molecular distinct subtypes on the clinicopathological findings of breast cancer stem cells in the Saudi population, the sample size is very limited. It was challenging to perform a subgroup analysis of genetic changes from each origin due to the limited data. Another limitation is the absence and/or limited data for the prognosis analysis. A study with a large number of cases from multicenter with applicable information should be conducted for future prospective to bypass these limitations.

To the best of our knowledge, we presented here the first-time results of next-generation sequencing in breast cancer patients in Saudi populations by applying immunohistochemistry evaluation of stem cell markers then applying NGS to detect different variations of TP53 gene. Due to insufficient information about the genetics of breast cancer in the Saudi population and limited resources for supporting the current study, the number of studies patients were small.

With the current findings, we are aiming to gain insight for a better understanding of breast cancer in the Saudi population. It is important to select appropriate patients with TP53 mutation to use anti-p53 therapies and ensure successful results in clinical studies. Accurate detection of TP53 mutation would be necessary using next-generation sequencing (NGS) which allows whole genomic evaluation. It is still impractical in most clinical laboratories to use NGS to determine the p53 mutational state due to high cost and complex interpretation. By contrast, IHC is still considered a valuable diagnostic tool with easy accessibility and well-established efficacy in investigating target agents and predicting clinical outcome in cancer [3].

\section{CONCLUSION}

We believe that the combination of CD44, CD24, and TP53 and TP53 gene mutation can be used as prognostic factors for breast cancer patients and the obtained information may also contribute to the development of a treatment.

\section{ACKNOWLEDGEMENTS}

The authors gratefully acknowledge the Institute of Scientific Research and Revival of Islamic Heritage and Deanship of Scientific Research, Umm Al-Qura University (Project No. 43509003) for the financial support.

\section{Contribution of Authors}

Hanan Abd ElMoneim and Ghada Esheba were involved in the design of the histopathology study and the selection of the cases. Rami Nassir and John Robbins were involved in the design of the molecular study and the selection of the cases Leimia Suliman was involved in the selection of the cases. Rami Nassir, Hanan Abd ElMoneim, Ghada Esheba, and John 
Robbins have contributed equally to the attainment, statistical analysis and interpretation of data; drafting and critically revising the article, and final approval of the manuscript.

\section{REFERENCES}

1. Siegel, R. L., Miller, K. D., \& Jemal, A. (2016). Cancer statistics, 2016. CA Cancer J Clin, 66(1), 7-30. doi:10.3322/caac. 21332

2. Al-Qasem, A. J., Toulimat, M., Eldali, A. M., Tulbah, A., Al-Yousef, N., Al-Daihan, S. K., . . . Aboussekhra, A. (2011). TP53 genetic alterations in Arab breast cancer patients: Novel mutations, pattern and distribution. Oncol Lett, 2(2), 363-369. doi:10.3892/ol.2011.236

3. Li, J. P., Zhang, X. M., Zhang, Z., Zheng, L. H., Jindal, S., \& Liu, Y. J. (2019). Association of p53 expression with poor prognosis in patients with triple-negative breast invasive ductal carcinoma. Medicine (Baltimore), 98(18), e15449. doi:10.1097/MD.0000000000015449

4. Alotaibi, R. M., Rezk, H. R., Juliana, C. I., \& Guure, C. (2018). Breast cancer mortality in Saudi Arabia: Modelling observed and unobserved factors. PLoS One, 13(10), e0206148. doi:10.1371/journal.pone.0206148

5. Merdad, A., Gari, M. A., Hussein, S., Al-Khayat, S., Tashkandi, H., Al-Maghrabi, J., . . Dallol, A. (2015). Characterization of familial breast cancer in Saudi Arabia. BMC Genomics, 16 Suppl 1, S3. doi:10.1186/1471-2164-16-S1-S3

6. Herzallah, H. K., Antonisamy, B. R., Shafee, M. H., \& Al-Otaibi, S. T. (2019). Temporal trends in the incidence and demographics of cancers, communicable diseases, and non-communicable diseases in Saudi Arabia over the last decade. Saudi Med J, 40(3), 277-286. doi:10.15537/smj.2019.3.23585

7. Qiu, Y., Pu, T., Guo, P., Wei, B., Zhang, Z., Zhang, H., . . . Ye, F. (2016). ALDH(+)/CD44(+) cells in breast cancer are associated with worse prognosis and poor clinical outcome. Exp Mol Pathol, 100(1), 145-150. doi:10.1016/j.yexmp.2015.11.032

8. Dittmer, J. (2018). Breast cancer stem cells: Features, key drivers and treatment options. Seminars in cancer biology, 53, 59-74. doi:10.1016/j.semcancer.2018.07.007

9. Jaggupilli, A., \& Elkord, E. (2012). Significance of CD44 and CD24 as cancer stem cell markers: an enduring ambiguity. Clin Dev Immunol, 2012, 708036. doi:10.1155/2012/708036

10. Tiezzi, D. G., Valejo, F. A., Marana, H. R., Carrara, H. H., Benevides, L., Antonio, H. M., . . . de Andrade, J. M. (2012). CD44+/CD24- cells and lymph node metastasis in stage I and II invasive ductal carcinoma of the breast. Med Oncol, 29(3), 1479-1485. doi:10.1007/s12032-011-0014-X

11. Bodian, D. L., McCutcheon, J. N., Kothiyal, P., Huddleston, K. C., Iyer, R. K., Vockley, J. G., \&
Niederhuber, J. E. (2014). Germline variation in cancer-susceptibility genes in a healthy, ancestrally diverse cohort: implications for individual genome sequencing. PLoS One, 9(4), e94554. doi:10.1371/journal.pone.0094554

12. Schon, K., \& Tischkowitz, M. (2018). Clinical implications of germline mutations in breast cancer: TP53. Breast Cancer Res Treat, 167(2), 417-423. doi:10.1007/s10549-017-4531-y

13. Lin, Y., Zhong, Y., Guan, H., Zhang, X., \& Sun, Q. (2012). CD44+/CD24- phenotype contributes to malignant relapse following surgical resection and chemotherapy in patients with invasive ductal carcinoma. J Exp Clin Cancer Res, 31, 59. doi:10.1186/1756-9966-31-59

14. Pfister, N. T., \& Prives, C. (2017). Transcriptional Regulation by Wild-Type and Cancer-Related Mutant Forms of p53. Cold Spring Harb Perspect Med, 7(2). doi:10.1101/cshperspect.a026054

15. Fukushima, S., Endo, M., Matsumoto, Y., Fukushi, J. I., Matsunobu, T., Kawaguchi, K. I., . . . Nakashima, Y. (2017). Hypoxia-inducible factor 1 alpha is a poor prognostic factor and potential therapeutic target in malignant peripheral nerve sheath tumor. PLoS One, 12(5), e0178064. doi:10.1371/journal.pone.0178064

16. Surbhi, Metgud, R., Naik, S., \& Patel, S. (2017). Spindle cell lesions: A review on immunohistochemical markers. $J$ Cancer Res Ther, 13(3), 412-418. doi:10.4103/09731482.176178

17. Hammond, M. E., Hayes, D. F., \& Wolff, A. C. (2011). Clinical Notice for American Society of Clinical Oncology-College of American Pathologists guideline recommendations on ER/PgR and HER2 testing in breast cancer. J Clin Oncol, 29(15), e458. doi:10.1200/JCO.2011.35.2245

18. Wolff, A. C., Hammond, M. E., Hicks, D. G., Dowsett, M., McShane, L. M., Allison, K. H., . . . College of American, P. (2014). Recommendations for human epidermal growth factor receptor 2 testing in breast cancer: American Society of Clinical Oncology/College of American Pathologists clinical practice guideline update. Arch Pathol Lab Med, 138(2), 241-256. doi:10.5858/arpa.2013-0953-SA

19. Wilson, C. A., Ramos, L., Villasenor, M. R., Anders, K. H., Press, M. F., Clarke, K., . . . Slamon, D. J. (1999). Localization of human BRCA1 and its loss in high-grade, non-inherited breast carcinomas. Nat Genet, 21(2), 236-240. doi: $10.1038 / 6029$

20. Chen, Y., Song, J., Jiang, Y., Yu, C., \& Ma, Z. (2015). Predictive value of CD44 and CD24 for prognosis and chemotherapy response in invasive breast ductal carcinoma. Int J Clin Exp Pathol, $8(9), 11287-11295$.

21. Bai, J., Chen, W. B., Zhang, X. Y., Kang, X. N., Jin, L. J., Zhang, H., \& Wang, Z. Y. (2020). HIF- 
2alpha regulates CD44 to promote cancer stem cell activation in triple-negative breast cancer via PI3K/AKT/mTOR signaling. World J Stem Cells, 12(1), 87-99. doi:10.4252/wjsc.v12.i1.87

22. Idowu, M. O., Kmieciak, M., Dumur, C., Burton, R. S., Grimes, M. M., Powers, C. N., \& Manjili, M. H. (2012). CD44(+)/CD24(-/low) cancer stem/progenitor cells are more abundant in triplenegative invasive breast carcinoma phenotype and are associated with poor outcome. Hum Pathol, 43(3), 364-373. doi:10.1016/j.humpath.2011.05.005

23. Da Cruz Paula, A., Leitao, C., Marques, O., Rosa, A. M., Santos, A. H., Rema, A., . . . Lopes, C. (2017). Molecular characterization of CD44(+)/CD24(-)/Ck(+)/CD45(-) cells in benign and malignant breast lesions. Virchows Arch, 470(3), 311-322. doi:10.1007/s00428-017-2068-4

24. Sheridan, C., Kishimoto, H., Fuchs, R. K., Mehrotra, S., Bhat-Nakshatri, P., Turner, C. H., . . . Nakshatri, H. (2006). CD44+/CD24- breast cancer cells exhibit enhanced invasive properties: an early step necessary for metastasis. Breast Cancer Res, 8(5), R59. doi:10.1186/bcr1610

25. Mansoori, M., Roudi, R., Abbasi, A., Abolhasani, M., Abdi Rad, I., Shariftabrizi, A., \& Madjd, Z. (2019). High GD2 expression defines breast cancer cells with enhanced invasiveness. Exp Mol Pathol, 109, 25-35. doi:10.1016/j.yexmp.2019.05.001

26. Jang, M. H., Kang, H. J., Jang, K. S., Paik, S. S., \& Kim, W. S. (2016). Clinicopathological analysis of CD44 and CD24 expression in invasive breast cancer. Oncol Lett, 12(4), 2728-2733. doi:10.3892/ol.2016.4987

27. Wang, Z., Wang, Q., Wang, Q., Wang, Y., \& Chen, J. (2017). Prognostic significance of CD24 and CD44 in breast cancer: a meta-analysis. Int $J$ Biol Markers, 32(1), e75-e82. doi:10.5301/jbm.5000224

28. Shokouh, T. Z., Ezatollah, A., \& Barand, P. (2015). Interrelationships Between Ki67, HER2/neu, p53, ER, and PR Status and Their Associations With Tumor Grade and Lymph Node Involvement in Breast Carcinoma Subtypes: RetrospectiveObservational Analytical Study. Medicine (Baltimore), 94(32), e1359. doi:10.1097/MD.0000000000001359

29. Abubakar, M., Guo, C., Koka, H., Sung, H., Shao, N., Guida, J., . . . Yang, X. R. (2019). Clinicopathological and epidemiological significance of breast cancer subtype reclassification based on p53 immunohistochemical expression. NPJ Breast Cancer, 5, 20. doi:10.1038/s41523-019-0117-7.

30. Fountzilas, G., Giannoulatou, E., Alexopoulou, Z., Zagouri, F., Timotheadou, E., Papadopoulou, K., . . . Kotoula, V. (2016). TP53 mutations and protein immunopositivity may predict for poor outcome but also for trastuzumab benefit in patients with early breast cancer treated in the adjuvant setting. Oncotarget, $\quad 7(22), \quad 32731-32753$. doi:10.18632/oncotarget.9022

31. Zhang, Y., Xiong, S., Liu, B., Pant, V., Celii, F., Chau, G., . . . Lozano, G. (2018). Somatic Trp53 mutations differentially drive breast cancer and evolution of metastases. Nat Commun, 9(1):3953. doi:10.1038/s41467-018-06146-9

32. Solomon, H., Dinowitz, N., Pateras, I. S., Cooks, T., Shetzer, Y., Molchadsky, A., . . . Rotter, V. (2018). Mutant p53 gain of function underlies high expression levels of colorectal cancer stem cells markers. Oncogene, 37(12):1669-1684. 